\title{
Evaluation of wavelength shifters for spectral separation of barium fluoride emissions
}

\author{
T.A. DeVol ${ }^{\mathrm{a}, *}$, D.K. Wehe ${ }^{\mathrm{b}}$, G.F. Knoll ${ }^{\mathrm{b}}$ \\ ${ }^{a}$ Environmental Systems Engineering Department, Clemson University, Anderson, SC 29625, USA \\ ${ }^{b}$ Department of Nuclear Engineering, The University of Michigan, Ann Arbor, MI 48109, USA
}

Received 15 March 1994

\begin{abstract}
Application of the barium fluoride $\left(\mathrm{BaF}_{2}\right)$ scintillator for characterization of intense radiation fields has been pursued since the fast component was discovered in the early 1980's. Unfortunately, $\sim 80 \%$ of the scintillator emissions have a slow $\sim 600$ ns decay time constant. The long decay time hampers the use of $\mathrm{BaF}_{2}$ at high count rates because the slow emissions appear as an afterglow which has an intensity that varies with the interaction rate in the crystal. The temporal separation of $\mathrm{BaF}_{2}$ emissions by spectral separation using wavelength shifting techniques has been explored in this work. Of the wavelength shifting techniques that were examined, the solvent excitation technique showed the greatest potential, but was insufficient by itself to suppress the slow emissions to the desired degree. All the wavelength shifting techniques yield an enhanced ratio of fast to slow photons, but a decreased fast photoelectron yield.
\end{abstract}

\section{Introduction}

Barium fluoride $\left(\mathrm{BaF}_{2}\right)$ scintillation was discovered in the early 1970 's [1]. The light output was measured to be $\sim 10 \%$ that of $\mathrm{NaI}(\mathrm{Tl})$ and to have a single $(630 \mathrm{~ns})$ decay time constant. Over a decade later, a fast $(0.8 \mathrm{~ns})$ decay time constant $[2,3]$ was discovered. The total luminosity of $\mathrm{BaF}_{2}$ is $\sim 20 \%$ that of $\mathrm{NaI}(\mathrm{Tl})$, with $13 \%$ of the $\mathrm{BaF}_{2}$ emissions (1400 photons / MeV [4]) having a 0.8 ns decay time constant. For high count rate applications, it may be advantageous to collect only the fast photons while avoiding the slow. Several researchers have shown reasonable separation of the $\mathrm{BaF}_{2}$ emission components using various techniques. Separation of the fast and slow components has been attempted using fast gated integration [3], solar blind photomultiplier tubes (PMT) [5,6], band-pass filtering [7], doping the crystal with lanthanum $[8,9]$, and by elevating the crystal temperature [10,11]. For applications utilizing the total light output of $\mathrm{BaF}_{2}$, wavelength shifters (WLSs) have been used to shift $\mathrm{BaF}_{2}$ emissions above the cut-off wavelength of borosilicate glass thus eliminating the need for a quartz endwindow PMT. The WLS was either vacuum evaporated onto the face of a scintillator [12-14], or incorporated into a polymer $[15,16]$ or liquid matrix [17]. However, WLSs have not been used to spectrally separate $\mathrm{BaF}_{2}$ emissions.

Fig. 1 shows the scintillation decay time spectrum of $\mathrm{BaF}_{2}$. The fast 0.8 ns (associated with cross-over luminescence) and the slow 578 ns (associated with self-trapped

* Corresponding author. exciton luminescence) decay time constants are apparent. Fig. 2 shows the emission spectrum of $\mathrm{BaF}_{2}$ with the distribution associated with the cross-over luminescence $\left(\lambda_{\text {peak }}=220,195 \mathrm{~nm}\right)$ and the self-trapped exciton luminescence $\left(\lambda_{\text {peak }}=300 \mathrm{~nm}\right)$. Note that by setting a discriminator at $\sim 240 \mathrm{~nm}$, a separation of the emissions in energy (wavelength) is also an approximate separation of the emission components with different fluorescence decay times. So by spectrally separating the emissions, one will approximately separate the various decay times. In this paper we will discuss the use of WLSs to spectrally separate $\mathrm{BaF}_{2}$ emissions, as a means to temporally separate them.

\section{Methodology}

There are two general techniques available for spectrally separating the $\mathrm{BaF}_{2}$ emissions using WLSs: orthogonal (see Fig. 3) and in-line (see Fig. 4) geometries. This paper primarily investigates two variations of the in-line technique which can be employed as either a solvent or solute excitation system. The orthogonal technique, which was developed by Lorenz et al. [18], is included for completeness. Both techniques are described in section 3 .

\subsection{Sample preparation}

The solvents were obtained from Aldrich Chemical Corporation and Fisher Scientific, and were HPLC (high purity liquid chromatography) or spectrophotometric grade (the purest commercially available grade for each com- 


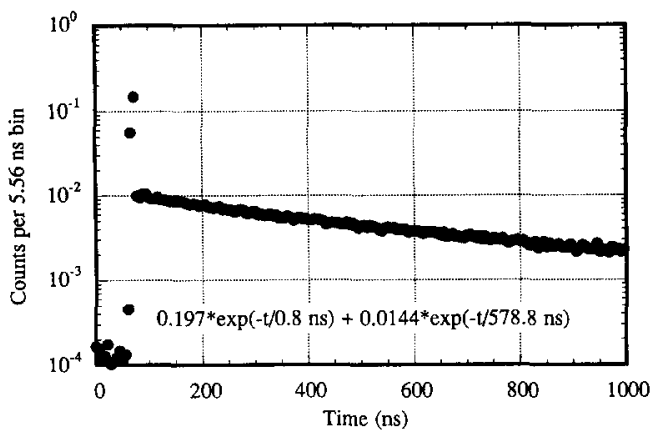

Fig. 1. Scintillation decay spectrum of $\mathrm{BaF}_{2}[17]$.

pany). The fluors were obtained from Aldrich Chemical Corporation (typical purity $99+\%$ ) and Lambda Physik, Inc (unknown purity). All chemicals were used as delivered without further purification. However, the solvents were de-oxygenated by bubbling dry nitrogen through them for at least $15 \mathrm{~min}$ and solutions were stored under dry nitrogen until measured. In most cases, the cuvettes and optical lenses were made of high purity quartz. Also, the long pass filter used to demonstrate the various WLS techniques was obtained from Oriel Corporation and was classified as "low-fluorescence". The $\mathrm{BaF}_{2}$ crystals (2.54 $\mathrm{cm}$ diam $\times 1 \mathrm{~cm}$ thick right circular cylinder) were obtained from Optovac, Inc.

\subsection{Sample geometry}

The WLS samples were liquids contained in a quartz glassware. For the orthogonal measurements, the WLS was contained in a square $1 \mathrm{~cm} \times 1 \mathrm{~cm}$ cuvette. The edges of the cuvette were masked during measurement to reduce the contribution of internally reflected $\mathrm{BaF}_{2}$ emissions from the sides of the cuvette. For the in-line solute excitation measurements the cuvette was $2.54 \mathrm{~cm}$ diam $\times 1 \mathrm{~cm}$ thick. The cuvettes allowed for easy exchange of the WLS. For

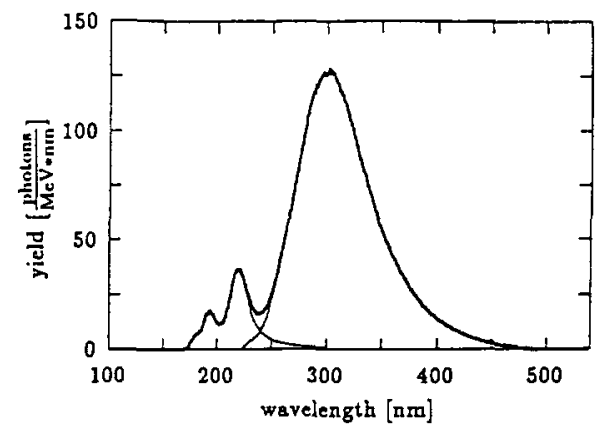

Fig. 2. $\mathrm{BaF}_{2}$ time resolved emission spectrum. Distributions of the cross-over luminescence ( $\lambda_{\text {peak }}=220,195 \mathrm{~nm}$; fast emissions) and self-trapped excitons ( $\lambda_{\text {peak }}=300 \mathrm{~nm}$; slow emissions) are shown [4].

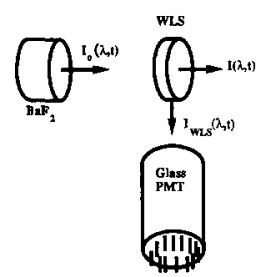

Fig. 3. Schematic diagram of the detection geometry for the orthogonal measurement.

the in-line solvent excitation technique, the WLS was contained between two glass slides that were separated by a thin gold foil. The foil assisted in containing the liquid WLS as well as serving as a spacer which defined the sample thickness.

\subsection{Properties of WLSs needed for spectral separation}

There are four primary properties of the WLS desired for applications involving $\mathrm{BaF}_{2}$ emissions: fluorescence at or near the peak spectral response of the photodetector, high fluorescence quantum efficiency, fast decay time constant, and absorption characteristics tailored to the $\mathrm{BaF}_{2}$ emission spectrum. Because the spectral response of a PMT is limited, it is advantageous that the solute fluorescence at $350-450 \mathrm{~nm}$ to maximize spectral matching between the PMT sensitivity and the solute fluorescence emission. Fluorescence quantum efficiency is as important as the fluorescence emission wavelength because it also influences the number of photons that will be detected. To assure negligible degradation in the signal, the fluorescence quantum efficiency $\left(Q_{\mathrm{f}}\right)$ should be as high as possible, e.g., $Q_{\mathrm{f}}>0.5$. The property that makes $\mathrm{BaF}_{2}$ of so much interest is the fast fluorescence decay time. For an application that includes high count rates, signal speed is of utmost importance. When wavelength shifting, the speed at which the signal can be acquired is dictated by the WLS decay time constant $(\tau)$. Therefore, it is advantageous to utilize a fluor that is as fast as possible, e.g., $\tau<10 \mathrm{~ns}$. Optical absorption was also studied in detail. The fluorescence emission, quantum efficiency and the decay time constant are not sensitive to the particular wavelength shifting technique used. However, the overall efficiency of the WLS in separating the $\mathrm{BaF}_{2}$ emissions depends strongly on the absorption characteristics for a given WLS tech-

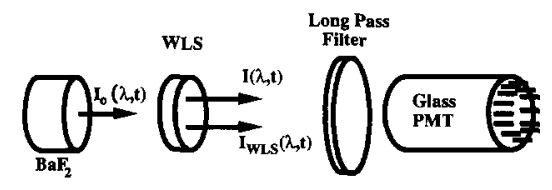

Fig. 4. Schematic diagram of the detection geometry for an in-line measurement. 
nique. To best describe these absorption characteristics, each WLS technique will be discussed separately in the next section.

\subsection{Measurements}

Several measurements were made to determine the usefulness of particular compound(s) for the spectral separation of $\mathrm{BaF}_{2}$ emissions. Absorption, fluorescence wavelength, quantum efficiency, and decay times of various WLSs were measured as well as the photoelectric yield measured by a borosilicate PMT when the WLS was coupled to the $\mathrm{BaF}_{2}$ crystal. The details of these measurements can be found elsewhere [17]. Organic molecular photophysics dictates the class of compounds that are likely to produce the desired wavelength shifting $\# 1$. Numerous candidate compounds were investigated for potential use. Of these compounds, only a few will be presented in section 3. For a complete listing of the compounds investigated, their properties and limitations in temporal separation, the reader is referred to DeVol [19].

\subsection{General considerations}

The objective of all the wavelength shifting techniques that are presented here is to spectrally separate $\mathrm{BaF}_{2}$ emissions by minimizing the detection of the emissions between $240<\lambda \leq 400 \mathrm{~nm}$ and maximizing the detection for $\lambda \leq 240 \mathrm{~nm}$ emissions. $240 \mathrm{~nm}$ is chosen as the cut-off wavelength because it is the optimum wavelength for temporal separation (refer to Fig. 2). The parameter of importance in this work is the ratio of fast to slow photoelectrons. This ratio can either be measured in time or energy (wavelength). For comparison, $R_{\mathrm{BaF} 2}(t)$ for bare $\mathrm{BaF}_{2}$ is calculated in the time domain as:

$$
R_{\mathrm{BaF}_{2}}(t)=\frac{\int_{0}^{\infty} I_{\text {fast }}(t) \mathrm{d} t}{\int_{0}^{\infty} I_{\text {slow }}(t) \mathrm{d} t}=0.20,
$$

or $R_{\mathrm{BaF} 2}(\lambda)$ can be evaluated in the energy (wavelength) domain as:

$$
R_{\mathrm{BaF}_{2}}(\lambda)=\frac{\int_{180 \mathrm{~nm}}^{240 \mathrm{~nm}} I_{\mathrm{BaF} 2}(\lambda) \mathrm{d} \lambda}{\int_{240 \mathrm{~nm}}^{400 \mathrm{~nm}} I_{\mathrm{BaF} 2}(\lambda) \mathrm{d} \lambda}=0.13 .
$$

$I_{\text {fast }}(t)$ and $I_{\text {slow }}(t)$ are determined from the functional form of the temporal distribution presented in Fig. 1. $I_{\mathrm{BaF} 2}(\lambda)$ is the background-subtracted, nonlinearity corrected emission spectrum presented in Fig. 2. Noting the overlap between the fast and slow emissions as shown in

\#1 For a description of the photophysical properties that limit the degree of spectral separation, the reader is referred to DeVol [19] and the references therein.
Fig. 2, the assertion that spectral separation also results in a temporal separation is not strictly correct which can also be shown by comparing the values of $R_{\mathrm{BaF}_{2}}(t)$ and $R_{\mathrm{BaF}_{2}}(\lambda)$. The goal is to minimize the contribution of the slow component coming from the $\mathrm{BaF}_{2}$ while efficiently shifting the fast component to a wavelength in the peak spectral range of the PMT. The figure of merit, $R$, for how well a WLS accomplishes spectral separation can be derived using $t$ or $\lambda$, i.e. $R(t)$ and $R(\lambda)$, respectively. Because of the spectral overlap of the fast and slow emissions of $\mathrm{BaF}_{2}, R(t)$ is not equal to $R(\lambda)$. However, because the overlap is relatively minor, either $R(t)$ or $R(\lambda)$ is sufficient to make quantitative comparisons of the relative effectiveness of various WLS. In most cases it is easier to evaluate $R(\lambda)$ than $R(t)$. While $R(\lambda)$ was used as a tool to evaluate a compound for spectral separation, $R(t)$ is our ultimate quantity of interest.

\section{Results and discussion}

\subsection{Orthogonal geometry}

As shown in Fig. 3, the orthogonal geometry technique is named for the geometrical arrangement of the scintillator/WLS and the PMT. Using this detection geometry, the photon intensity emitted from the $\mathrm{BaF}_{2}$ crystal, $I_{0}(\lambda, t)$, is incident on the WLS. The transmitted photon intensity, $I(\lambda, t)^{\# 2}$, is a measure of the photons that pass through the WLS without absorption. Of those photons that are absorbed, some are absorbed by the solute which will fluoresce with probability $Q_{\mathrm{f}}$. In the orthogonal technique, a solute is chosen such that $\lambda_{\max }$ (the wavelength above which $\varepsilon(\lambda)=0$ ) is as low as practical. Generally, as $\lambda_{\max }$ decreases $\tau$ increases, and $Q_{\mathrm{f}}$ decreases [19]. The maximum value of $R(\lambda)$ is achieved with the compounds that typically have the longer $\tau$ and the smaller $Q_{\mathrm{f}}$ therefore, trade-offs have to be made between optimizing $R(\lambda)$, and $\tau$ and $Q_{\mathrm{f}}$, simultaneously. The optimum balance between $R(\lambda)$, and $Q_{\mathrm{f}}$ and $\tau$ seems to exist in the terphenyl group of the p-oligophenylene series. Fig. 5 summarizes the absorption and fluorescence of $2,2^{\prime \prime}$ diphenyl-p-terphenyl (BMT), a compound from the terphenyl group. Because the BMT emissions occur in the emission band of $\mathrm{BaF}_{2}$, geometrical discrimination rather than wavelength discrimination must be used to separate the various emission components. By placing the PMT orthogonal to the axis of the $\mathrm{BaF}_{2}$ crystal and WLS, minimum transmitted $\mathrm{BaF}_{2}$ photons will be detected. Unfortunately, only a small signal will be detected in the orthogonal technique because of the low light collection efficiency of the wavelength

\#2 The intensity of a transmitted beam of photons, $I(\lambda, t)$, is given as, $I(\lambda, t)=I_{0}(\lambda, t) 10^{-\varepsilon(\lambda) b x}$, where $\varepsilon(\lambda)$ is the molar extinction coefficient, $b$ is the molar concentration, $x$ is the thickness of the sample, and $I_{0}$ is the incident intensity. 


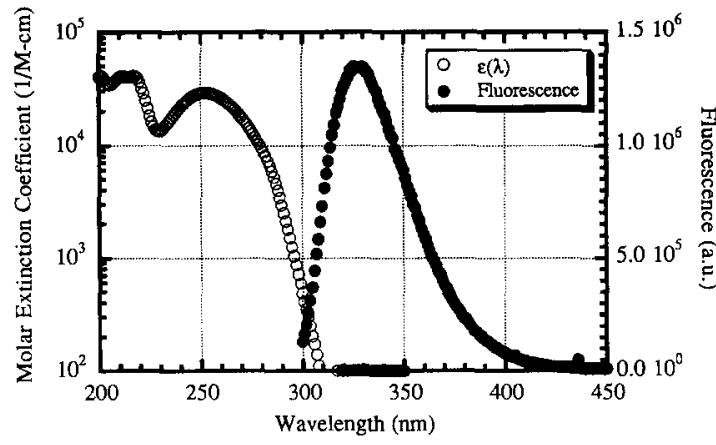

Fig. 5. Measured absorption and emission spectra of BMT.

shifted photons. Lorenz et al. [18] reported measuring a total of $50 \mathrm{PE} / \mathrm{MeV}$ using the orthogonal technique with BMT.

Using a time correlated single photon technique [17], $R(t)$ for BMT was determined to be 0.33 , thus resulting in a typical fast PE yield of $\sim 17 \mathrm{PE} / \mathrm{MeV} . R(t)$ is calculated using Eq. (1), where $I_{\text {fast }}(t)$ and $I_{\text {slow }}(t)$ are determined for the temporal distribution of the WLS when excited with $\mathrm{BaF}_{2}$ [17]. $R(t)$ can be compared to $R(\lambda)$ which is calculated in the following manner:

$R(\lambda)=\frac{\int_{180 \mathrm{~nm}}^{240 \mathrm{~nm}} I_{\mathrm{BaF}_{2}}(\lambda)\left(1-10^{-\varepsilon(\lambda) b x}\right) \mathrm{d} \lambda}{\int_{180 \mathrm{~nm}}^{400 \mathrm{~nm}^{2}} I_{\mathrm{BaF}_{2}}(\lambda)\left(1-10^{-\varepsilon(\lambda) b x}\right) \mathrm{d} \lambda}$

where $1-10^{-\varepsilon(\lambda) b x}$ is an approximation to the absorption probability. As discussed in the next section, a more useful quantity for evaluation is $R_{98 \%}(\lambda) . R_{98 \%}(\lambda)$ is the ratio of the wavelength shifted fast to slow signal that would be expected if $98 \%$ of the fast photons that are incident on the WLS are absorbed, which is calculated in the following manner:

$$
R_{98 \%}(\lambda)=\frac{0.98 \int_{180 \mathrm{~nm}}^{240 \mathrm{~nm}} I_{\mathrm{BaF}_{2}}(\lambda) \mathrm{d} \lambda}{\int_{180 \mathrm{~nm}}^{400 \mathrm{~nm}} I_{\mathrm{BaF}_{2}}(\lambda)\left(1-10^{-\varepsilon(\lambda) b x}\right) \mathrm{d} \lambda} .
$$

Table 1 summarizes the pertinent values of the most promising compounds that were studied for the orthogonal technique.

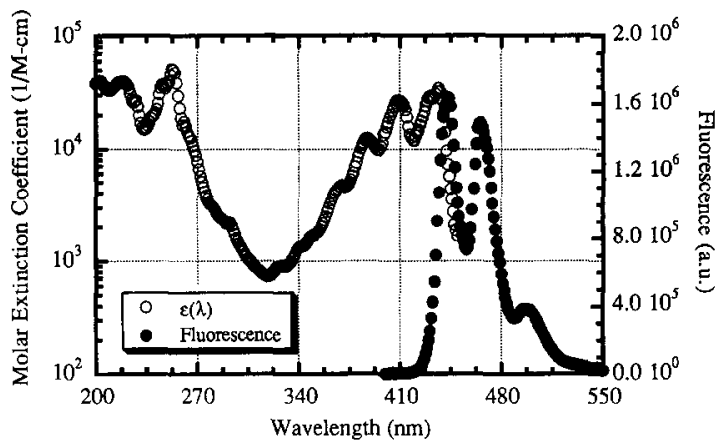

Fig. 6. Measured absorption and fluorescence emission spectra of perylene.

\subsection{In-line geometry}

The concept of maximum detection of the emissions below $240 \mathrm{~nm}$ combined with minimum detection of the emissions above $240 \mathrm{~nm}$ is the same for the in-line techniques as in the orthogonal technique, but the method to discriminate between the $\mathrm{BaF}_{2}$ and WLS photons is different. Fig. 4 illustrates the detection geometry used. The WLS used in the in-line geometry technique are such that the emission wavelength is higher than the $\mathrm{BaF}_{2}$ emissions. By introducing an appropriate long-pass filter between the WLS and PMT, the photons that are measured are primarily those that have been wavelength shifted. An appropriate long-pass filter is one that has maximum absorption of the incident $\mathrm{BaF}_{2}$ emissions and minimum absorption of the WLS emissions. The advantage of the in-line geometry over the orthogonal geometry is that there is higher light collection efficiency in the former. The choice between emphasizing solute and solvent excitation is an inherent difference in the wavelength shifting system that is used.

\subsubsection{Solute excitation}

The in-line solute excitation technique places a strong emphasis not only on the magnitude of the fluor molar extinction coefficient, $\varepsilon(\lambda)$, but on its shape as well. The principle behind the technique involves choosing a fluor that has minimum absorption, i.e. minimum $\varepsilon(\lambda)$, corresponding to the maximum $\mathrm{BaF}_{2}$ emission wavelength (300

Table 1

Results of orthogonal technique

\begin{tabular}{llllllll}
\hline Compound & $\lambda_{\mathrm{f}}[\mathrm{nm}]$ & $\mathrm{Q}_{\mathrm{f}}$ & $\tau[\mathrm{ns}]$ & $R_{98 \%}(\lambda)$ & $R(\lambda)$ & $R(t)$ & $\begin{array}{l}\text { Fast PE } \\
\text { yield }^{\mathrm{a}}\end{array}$ \\
\hline BMT $^{\mathrm{b}}$ & 330 & & 0.58 & 1.09 & 0.42 & 0.24 & 0.33 \\
PTP $^{\mathrm{c}}$ & 340 & 0.69 & 1.14 & 0.21 & 0.19 & 0.27 & 17 \\
\hline
\end{tabular}

\footnotetext{
a Fast PE yield per MeV of energy deposited is based on $R(t)$, data obtained from Lorenz [19] and measured values of $Q_{\mathrm{f}}$.

b $2,2^{\prime \prime}$ Dimethyl-p-terphenyl $(1.0 \mathrm{~g} / 1)$ cyclohexane.

c p-Terphenyl $(0.5 \mathrm{~g} / 1)$ cyclohexane.
} 


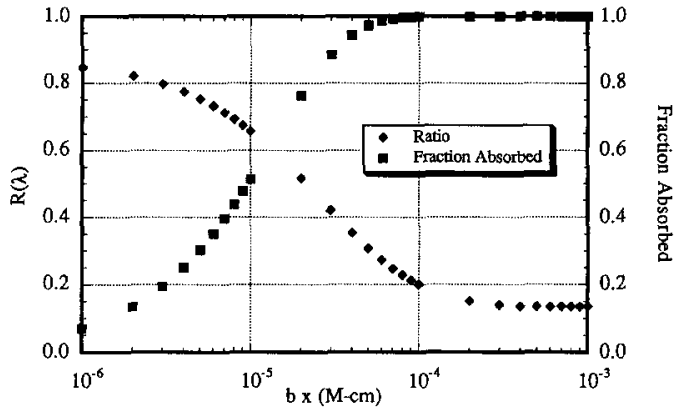

Fig. 7. $R(\lambda)$ and the probability of absorption of the $\mathrm{BaF}_{2}$ fast emissions for perylene dissolved in cyclohexane as a function of solute number density.

$\mathrm{nm}$ ) and the width of the "valley" of the absorption spectrum that is as wide as the FWHM of the $\mathrm{BaF}_{2}$ slow emissions $(\sim 75 \mathrm{~nm})$. Fig. 6 depicts an example of an absorption spectrum of a fluor that could be used for in-line solute excitation. The "fast" $\mathrm{BaF}_{2}$ photons are to be "strongly" absorbed into the second and third excited singlet states while the "slow" photons are "weakly" absorbed into the first excited state of the solute compound.

Fig. 7 shows that as the solute number density $(b x)$ decreases the fraction of incident fast photons that are absorbed decreases, but the ratio of fast to slow $R(\lambda)$, increases. Because large values of $R(\lambda)$ and high absorption efficiency of the fast emissions are in competition with each other, a metric, $R_{98 \%}(\lambda)$, is used to evaluate the possible potential of a fluor. Table 2 summarizes a few of the fluors that were investigated for the in-line solute excitation technique. Of the fluors listed in Table 2, it is clear that with the exception of perylene, the highest value of $R_{98 \%}(\lambda)$ exists for those compounds having the lowest fast PE yield.

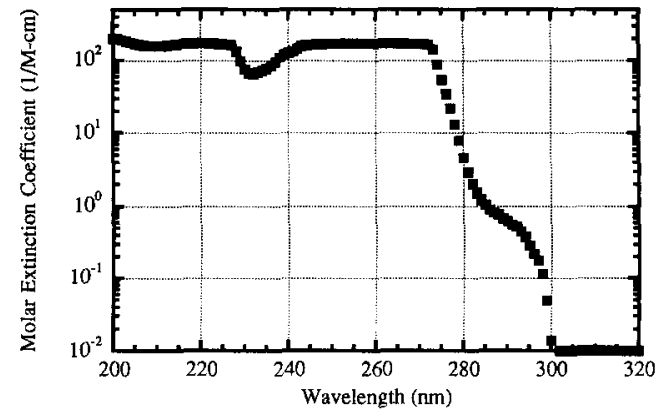

Fig. 8. Molar extinction coefficient of toluene.

\subsubsection{Solvent excitation}

Of the three techniques to spectrally separate $\mathrm{BaF}_{2}$ emissions using WLS techniques, in-line solvent excitation technique has the greatest potential of achieving the largest value of $R$ while delivering high efficiency and fast characteristic times. The solvent excitation technique is a combination of the best features of the orthogonal and in-line solute excitation techniques. In the previous techniques, the solute was directly excited by the UV emissions of $\mathrm{BaF}_{2}$, the solvent was present merely as a "'holder" of the solute molecules, any energy absorbed by the solvent was dissipated intramolecularly. In the in-line solvent excitation technique, the solvent plays an active role in the absorption of the incident energy. An example of a system that has appreciable energy transfer from the solvent to the solute, under the proper conditions, is 9,10 diphenyl anthracene (DPA) dissolved in toluene. Intermolecular energy transfer is dependent on having a sufficient concentration of solute such that the energy absorbed by the solvent can be very efficiently transferred to the solute. The excited solute molecule can then fluoresce with its high quantum efficiency and fast characteristic times.

Table 2

Results of in-line solute excitation technique

\begin{tabular}{lllllllc}
\hline Compound & $\lambda_{\mathrm{f}}[\mathrm{nm}]$ & $Q_{\mathrm{f}}$ & $\tau[\mathrm{ns}]$ & $R_{98 \%}(\lambda)$ & $R(\lambda)$ & $R(t)$ & $\begin{array}{l}\text { Fast PE } \\
\text { yield }^{\mathrm{a}}\end{array}$ \\
\hline DPA $^{\mathrm{b}}$ & 410 & 0.76 & 7.08 & 0.15 & 0.32 & 0.23 & 60 \\
Perylene $^{\mathrm{c}}$ & 445 & 0.53 & 5.94 & 0.29 & 0.50 & 0.32 & 52 \\
DOCI $^{\mathrm{d}}$ & 500 & 0.017 & 2.37 & 0.26 & 0.24 & 0.06 & 0.3 \\
DASBTI $^{\mathrm{e}}$ & 520 & 0.088 & & 0.27 & 0.29 & 0.3 & 13 \\
DCF f & 530 & 0.51 & 7.75 & 0.26 & 0.29 & 0.08 & \\
\hline
\end{tabular}

\footnotetext{
${ }^{a}$ Perylene fast PE yield per $\mathrm{MeV}$ was measured, the other values were scaled relative to the measured values of $Q_{\mathrm{f}}$.

b 9,10 Diphenyl anthracene $(0.01 \mathrm{~g} / 1)$ cyclohexane.

c Perylene $(0.01 \mathrm{~g} / 1)$ cyclohexane.

d 3,3'-Diethyloxacarbocyanine $(0.02 \mathrm{~g} / \mathrm{l})$ cyclohexane.

e 2(p-Dimethylaminostyrl)-benzothiazolylethyl $(0.02 \mathrm{~g} / \mathrm{l})$ cyclohexane.

f 2,7 Dichlorofluorescein $(0.02 \mathrm{~g} / 1)$ cyclohexane.
} 


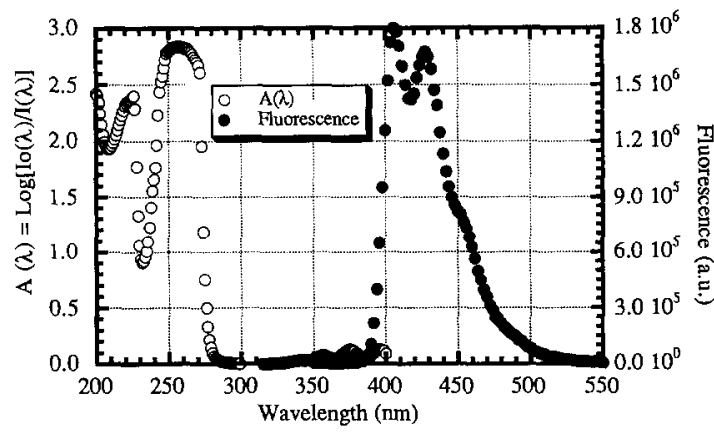

Fig. 9. Absorptivity (12.5 $\mu \mathrm{m}$ thick sample) and fluorescence of DPA dissolved at a concentration of $1 \mathrm{~g} / \mathrm{l}$ in toluene.

The largest value of $R_{98 \%}(\lambda)$ is achieved by selecting a good aromatic solvent with minimal absorption of the slow $\mathrm{BaF}_{2}$ emissions. The $\mathrm{BaF}_{2}$ emissions that are not absorbed by the solvent are absorbed by the long pass filter that is positioned between the WLS and PMT as illustrated in Fig. 4. As with the in-line solute excitation technique the filter is set to have maximum absorption of the $\mathrm{BaF}_{2}$ emissions and minimum absorption of WLS emissions.

Toluene, a well established efficient aromatic solvent, was chosen to demonstrate the in-line solvent excitation technique. As seen in Fig. 8, toluene begins to have appreciable absorption at $\sim 277 \mathrm{~nm}$. Very thin samples, e.g. $12.5 \mu \mathrm{m}$, were made to assure minimum direct absorption by the solute. Fig. 9 shows the absorptivity and fluorescence of DPA dissolved at a concentration of $1 \mathrm{~g} / \mathrm{l}$ in toluene. Note the apparent large Stokes shift that results from the solvent excitation technique. Some direct absorption by the solute exists and can be seen by the structure at $\sim 350-400 \mathrm{~nm}$, but the primary absorption is by the toluene. The fluorescence from the sample is quite clearly attributed to the DPA. As in the in-line solute excitation technique, $R(\lambda)$ and the probability of absorption are dependent on the sample thickness. The trends are the same as in the in-line solute excitation technique. The in-line solvent excitation technique results are summarized in Table 3.

Although this technique does offer the greatest potential for "large" $\boldsymbol{R}_{98 \%}(\lambda)$ and high efficiency wavelength shifting, $R$ is not as large as initially hoped. As was demonstrated with DPA dissolved in toluene at a thickness of $12.5 \mu \mathrm{m}$, if the thickness is decreased or if toluene is substituted with another efficient aromatic solvent with a lower absorption cut-off, then it may be possible to hypsochromically shift the absorption by $10 \mathrm{~nm}$, resulting in a
Table 4

Summary of techniques investigated and comparison to other techniques.

\begin{tabular}{llc}
\hline Technique & $R(\mathrm{t})$ & Fast PE Yield $^{2}$ \\
\hline Orthogonal & 0.33 & 17 \\
In-line: solute & 0.32 & 52 \\
In-line: solvent & 0.30 & 41 \\
$\mathrm{BaF}_{2}$ : Quartz & 0.20 & 186 \\
$\mathrm{BaF}_{2}$ : Borosilicate & 0.02 & 7 \\
\hline
\end{tabular}

${ }^{a}$ per $\mathrm{MeV}$

calculated value of $R_{98 \%}(\lambda)$ of 0.8 . Other researchers $[20,21]$ have shown that wavelength shifting efficiency of a thin film WLS can be greater than $90 \%$. Such a high WLS efficiency combined with the high light detection efficiency would result in a fast photoelectron yield that is higher than presented here.

\section{Conclusions}

The goal of this paper was to determine if spectral separation of $\mathrm{BaF}_{2}$ emissions using wavelength shifting techniques are an efficient and viable for the temporal separation of the emissions. $R(\lambda)$ and $R_{98 \%}(\lambda)$ were used as metrics to assess the ability of a WLS to perform spectral separation. Both $R(\lambda)$ and $R_{98 \%}(\lambda)$ are only approximations to $R(t)$, the important quantity, and the source of differences in these values have been discussed. A $\sim 50 \%$ increase in the fast to slow ratio (relative to $\mathrm{BaF}_{2}$ coupled to a quartz end-window PMT) was achieved using WLS. This was accomplished by a substantial decrease, however, in the fast PE yield. Table 4 summarizes $R(t)$ and the fast PE yield of the best data obtained for each technique as well as comparisons to $\mathrm{BaF}_{2}$ measured with a quartz and borosilicate endwindow PMT. The greatest potential for high efficiency spectral separation comes from the in-line geometry techniques. More work with the solvent excitation technique may be the best place to concentrate further efforts, particularly in evaluating more efficient, low wavelength cut-off aromatic solvents. If one of these solvents offers sufficient solubility and a thin sample is used, it may be possible to move the absorption cut-off as low as $260 \mathrm{~nm}$ and obtain a $R_{98 \%}(\lambda)$ of $\sim 0.8$. Unfortunately, as with the other techniques used to spectrally separate $\mathrm{BaF}_{2}$ emissions, wavelength shifting is lim-

Table 3

Properties and results on in-line solvent excitation technique

\begin{tabular}{|c|c|c|c|c|c|c|c|}
\hline Sample & $\lambda_{\mathrm{f}}[\mathrm{nm}]$ & $Q_{\mathrm{f}}$ & $\tau[\mathrm{ns}]$ & $R_{98 \%}(\lambda)$ & $R(\lambda)$ & $R(\mathrm{t})$ & Fast PE yield ${ }^{b}$ \\
\hline DPA/Toluene ${ }^{a}$ & 410 & 0.76 & 7.08 & 0.53 & 0.53 & 0.30 & 41 \\
\hline
\end{tabular}

${ }^{a}$ DPA $(1 \mathrm{~g} / 1)$ Toluene

${ }^{\mathrm{b}}$ per $\mathrm{MeV}$ 
ited by physical properties imposed by the particular technique.

Light collection efficiency imposed by the geometry arrangement reduced the $\mathrm{PE}$ yield. The measurements made with liquid WLS could be repeated under similar conditions using a polymer solvent. Use of polymers would alleviate the need for cuvettes and slides. In addition, a thin film of a non-fluorescent doped polymer could be used as a long-pass filter. Reducing the spacing between the PMT and the $\mathrm{BaF}_{2}$ will increase the light collection efficiency and thus increase the PE yield values beyond that are presented here. It is theoretically possible for the fast PE yield from a WLS to approach or exceed the fast $\mathrm{PE}$ yield of $\mathrm{BaF}_{2}$ coupled directly to a quartz endwindow PMT. The WLS fluoresces at a longer wavelength (lower energy) than the absorbed photons potentially resulting in better spectral matching between the fluor emissions and the spectral response of the PMT. If the losses imposed by the quantum efficiency and the geometry of the WLS are on the same order of magnitude as the gains in photoelectron yield resulting from better matching of the solute emission and PMT spectral response, then the photoelectron yield could remain the same or possibly increase over the $\mathrm{BaF}_{2}$ /quartz PMT values.

\section{Acknowledgements}

The authors wish to thank Dr. Raoul Kopelman for his assistance on the organic molecular photophysics, Dr. Mary Brake for the use of her monochromator, Dr. William W. Moses for his help in establishing our confidence in the determination of the time spectra, Dr. Stephen Derenzo for use of his nonlinear least squares fitting program, and John Valentine and Valentin Jordanov for their assistance in calibrating our MCA to units of charge. T.A. DeVol performed this research while under appointment to the Environmental Restoration and Waste Management Fellowship Program, which is administered by Oak Ridge
Associated Universities for the U.S. Department of Energy.

\section{References}

[1] M.R. Faruhki and C.F. Swinehart, IEEE Trans. Nucl. Sci. NS-18 (1971) 200.

[2] N.N. Ershov, N.G. Zakharov and P.A. Rodnyi, Opt. Spectrosc. 53 (1982) 51.

[3] M. Laval et al., Nucl. Instr. and Meth. 206 (1983) 169.

[4] P. Dorenbos, J.T.M. deHass, R. Visser, C.W.E. van Eijk and R.W. Hollander, IEEE Trans. Nucl. Sci. NS-40 (1993) 424.

[5] H. Kobayashi et al., Nucl. Instr. and Meth. A 270 (1988) 106.

[6] Z.Y. Wei, R.Y. Zhu, H. Newman and Z.W. Yin, Nucl. Instr. and Meth. B 61 (1991) 61.

[7] S. Shojiro et al., Nucl. Instr. and Meth. A 336 (1993) 179.

[8] P. Schotanus, P. Dorenbos, C.W.E. Van Eijk and H.J. Lamfers, Nucl. Instr. and Meth. A 281 (1989) 162.

[9] C.L. Woody, P.W. Levy and J.A. Kierstead, IEEE Trans. Nucl. Sci. NS-36 (1989) 536.

[10] J.H. Beaumont, W. Hayes, D.L. Kirk and G.P. Summers, Proc. Roy. Soc. Lond. A 315 (1970) 69.

[11] P. Schotanus, C.W.E. Van Eijk, R.W. Hollander and J. Pijpelink, Nucl. Instr. and Meth. 238 (1985) 564.

[12] E. Dafni, Nucl. Instr. and Meth. A 254 (1987) 54.

[13] W. Klamra, Th. Lindblad, M. Moszynski, L.O. Norlin and J. Weimer, Nucl. Instr. and Meth. A 265 (1988) 485.

[14] V. Yanovsky and P. Kozma, Nucl. Instr. and Meth. A 276 (1989) 659.

[15] E. Lorenz, G. Mageras and H. Vogel, Nucl, Instr. and Meth. A 249 (1986) 235.

[16] A.M. Gorin et al. Nucl. Instr. and Meth. A 251 (1986) 461.

[17] T.A. DeVol, D.K. Wehe and G.F. Knoll, Nucl. Instr. and Meth. A 327 (1993) 354.

[18] E. Lorenz, Max Plank Institut für Physik, private communication (1992).

[19] T.A. DeVol, Ph.D. Dissertation, The University of Michigan (1993).

[20] W. Viehmann and R.L. Frost, Nucl. Instr. and Meth. 167 (1979) 405.

[21] M. Grande, and G.R. Moss, Nucl. Instr. and Meth. 215 (1983) 539. 